\title{
Overexpression of filamin-A protein is associated with aggressive phenotype and poor survival outcomes in NSCLC patients treated with platinum-based combination chemotherapy
}

\author{
M. GACHECHILADZE ${ }^{1, *}$, J. SKARDA ${ }^{1 *}$, M. JANIKOVA ${ }^{1}$, G. MGEBRISHVILI ${ }^{1}$, G. KHARAISHVILI ${ }^{1}$, V. KOLEK², I. GRYGARKOVA², J. KLEIN ${ }^{3}$, \\ A. POPRACHOVA ${ }^{4}$, M. ARABULI ${ }^{5}$, Z. KOLAR ${ }^{1}$
}

\begin{abstract}
${ }^{1}$ Department of Clinical and Molecular Pathology, Institute of Molecular and Translational Medicine, Faculty of Medicine and Dentistry, Palacký University Olomouc, Czech Republic; ${ }^{2}$ Department of Tuberculosis and Respiratory Diseases, Faculty of Medicine and Dentistry, Palacký University Olomouc and University Hospital Olomouc; ${ }^{3}$ Laboratory of Experimental Medicine, Departments of Pediatrics and Oncology, Faculty of Medicine and Dentistry, Palacký University Olomouc and University Hospital Olomouc; ${ }^{4}$ Department of Normal Anatomy, Faculty of Medicine and Dentistry, Palacký University Olomouc; ${ }^{5}$ Department of Normal Anatomy, Tbilisi State Medical University, Tbilisi, Georgia
\end{abstract}

*Correspondence: jojos@email.cz, marjammg@gmail.com

Received February 24, 2015 / Accepted September 16, 2015

\begin{abstract}
An actin-binding protein filamin A connects the actin filament network to cell membrane receptors, and acts as a scaffold for various signaling pathways related to cancer growth and progression. Recently, it has been reported that filamin A is required for efficient regulation of early stages of DNA repair process. Moreover, some in vitro studies showed that the overexpression of filamin A determines resistance to various cytotoxic drugs, including cisplatin. We aimed to analyse the expression of filamin A protein in resected NSCLC (Non Small Cell Lung Cancer) specimens, to investigate the association of the level of filamin A protein expression and other clinicopathological features, and possible relationship between the expression of filamin A and survival outcome in NSCLC patients, treated with platinum-based combination chemotherapy. We performed filamin A protein immunohistochemistry on formalin-fixed and paraffin-embedded (FFPE) tissue sections from 135 NSCLC patients, using EP2405Y antibody against C-terminus of filamin A. Cytoplasmic, membranous and nuclear positivity of filamin A was evaluated semi-quantitatively and correlated with available clinicopathological data. Patients were divided into two groups for survival analysis (I group - patients treated with adjuvant platinum-based chemotherapy, II group - patients with surgical treatment only). We found significant positive correlation between filamin A protein expression and NSCLC stage $(\mathrm{r}=0.249 ; \mathrm{p}<0,05)$, presence of lymph node $(\mathrm{N})(\mathrm{r}=0.205 ; \mathrm{p}<0,05)$ and distant metastases $(\mathrm{M})(\mathrm{r}=0.332 ; \mathrm{P}<0.01)$. Increased filamin A protein expression was significantly related with poor survival outcomes in patients with adjuvant platinum-based chemotherapy: OS (HR=1.005, 95\%CI[1.000;1.010], $\mathrm{p}=0.037)$, DFS (HR=1.004, 95\%CI [1.001:1.008], $\mathrm{p}=0,017)$. Multivariate Cox proportional hazards regression analysis also showed that overexpression of filamin A represents an independent risk factor for disease relapse, in addition to tumor size, stage, and metastases status ( $\mathrm{HR}=1.723,95 \% \mathrm{CI}$ [1.021:2.909], $\mathrm{p}<0.05)$. Thus, filamin A expression might be a new prognostic marker in patients with NSCLC.
\end{abstract}

Key words: filamin A, non-small-cell lung cancer, prognosis, cisplatin, chemoresistance

Non-small cell lung cancer (NSCLC) accounts for 85\% of all lung cancer cases and represents the leading cause of cancer related mortality worldwide [1]. The most favourable outcome in patients with resected NSCLC in stage IB-III has been shown after platinum-based chemotherapy. However, the improvement of survival rate using this type of chemotherapy was only $\sim 5 \%$ [2]. In the majority of cases, treatment efficacy is markedly reduced by intrinsic or acquired chem- oresistance. Mechanisms of resistance to platinum-based compounds, such as cisplatin and carboplatin are complex and numerous, including the highly efficient DNA damage repair [3,4]. According to recent experimental studies, filamin A has emerged as one of the key proteins involved in the DNA damage response.

Filamin-A (also known as human actin-binding protein 280 (ABP-280) or filamin-1), is a cytoskeletal protein involved in 
regulation of cell shape and locomotion. The primary function of filamin A protein is to cross-link actin filaments into orthogonal networks and assist the formation of cytoskeleton. Filamin A also connects actin networks to plasma membrane and facilitates cell-matrix interactions. This aside, filamin A interacts with more than 60 non-cytosceletal proteins related to cancer growth and metastasis and, serves as a scaffold for various signaling pathways [5]. A number of studies report different levels of filamin-A expression in cancer tissue, compared to healthy tissue. Increased expression of filamin-A has been found in colorectal [6]], pancreatic [7], hepatic [8] and breast [9] carcinomas, whilst there is a marked decrease of filamin-A expression in human bladder [10] and nasopharyngeal [11] cancer.

Recently, it has been shown that filamin A protein plays an essential role in earlier stages of DNA repair via interaction with BRCA1 and BRCA2 in vitro $[12,13]$. The overexpression of filamin A determines resistance to various cytotoxic drugs, including bleomicin and cisplatin in melanoma cell lines and mouse xenograft models [14]. Also, A2780 epithelial ovarian cancer cell line, with acquired platinum resistance, shows markedly increased levels of filamin A mRNA [15]. These experimental data suggest the potential role of filamin A protein expression as a marker of DNA damaging chemotherapy response, however the clinical relevance of this information is lacking.

The aim of this study was to analyse the expression of filamin A protein in resected NSCLC specimens, to investigate the association of the level of filamin A protein and other clinicopathological features as well as possible relationships between the expression of filamin A and survival outcome of patients treated with platinum-based combination chemotherapy.

\section{Patients and methods}

Patient group and sample collection. The sample included 135 non-small cell lung cancer patients who were diagnosed at the University Hospital, Faculty of Medicine and Dentistry, Palacky University, Olomouc, Czech Republic, between 1996-2000 and 2003-2011. The patient selection was based on the availability of the clinical data. The patients were treated by radical surgery alone or radical surgery combined with adjuvant platinum-based chemotherapy (Carboplatin+Navelbin). Patients treated by neoadjuvant therapy or other than a platinum-based regimen were excluded from the study. Corresponding archival tissue samples were obtained from the archive of the Department of Clinical and Molecular Pathology, Faculty of Medicine and Dentistry, Palacky University. Patient samples were re-examined and clinicopathological/demographic parameters including age, gender, clinical stage, depth of tumour invasion, lymph node metastasis, distant metastasis and differentiation were obtained. The patients were categorised according to WHO classification of tumors of the lung (2004). The end-points of the study were determined as overall survival (OS) and disease-free survival (DFS). OS was defined as the period from the date of surgery until death or to the last date of follow-up; DFS was defined as the period from the date of surgery to the date of relapse or metastasis. Cases lost during follow-up and those ending in death from any cause other than lung cancer were regarded as censored data in the analysis of survival rates. Median follow-up was 38 months (range 2 - 181 months). Detailed clilnicopathological characteristics of patients and tumors are given in Table 1 .

Immunohistochemical staining. Sections $(4 \mu \mathrm{m})$ from formalin-fixed and paraffin-embedded (FFPE) tissue samples were used and stained by using Ventana Benchmark XT automatic tissue stainer from Roche. The monoclonal EP2405Y antibody against C-terminus of filamin A (LSBio) was used in dilution 1:500. Tissue sections without primary antibody addition and served as a negative control. Normal skin tissue was used as a positive control. The stained slides were blindly evaluated by two pathologists (M.G, J.Š). Membranous cytoplasmic staining was assesed by the histoscore method (percentage of positive cells $\mathrm{x}$ intensity of staining -1 , weak; 2 , moderate; and 3 , strong). The final histoscore ranged from 0 (minimum) to 300 (maximum)). Non-representative, dam-

Table 1. Patients and tumor characteristics

\begin{tabular}{llcc}
\hline CHARACTERISTICS & SUBGROUPS & N & $\%$ \\
\hline SEX & MALE & 103 & 76.3 \\
AGE & FEMALE & 32 & 23.7 \\
\multirow{4}{*}{ HISTOLOGY } & $\leq 60$ & 62 & 45.9 \\
& $>60$ & 73 & 54.1 \\
& Adenocarcinoma & 53 & 39.3 \\
& Squamous cell carcinoma & 66 & 48.9 \\
& Large cell carcinoma & 14 & 10.4 \\
GRADE & Adenosqamous & 2 & 1.5 \\
& carcinoma & & \\
& G1 & 15 & 11.1 \\
NODAL STATUS & G2 & 39 & 28.9 \\
& G3 & 81 & 60.0 \\
& NEGATIVE & 54 & 40 \\
DISTANT METASTASES & PRESENT & 70 & 51.9 \\
& NOT PRESENT & 11 & 8.1 \\
ADJUVANT & NOT SPECIFIED & 9 & 5.9 \\
STAGE & I & 116 & 85.9 \\
& II & 53 & 8.1 \\
& III & 26 & 19.3 \\
& IV & 47 & 34.8 \\
& YES & 9 & 6.7 \\
& NO & 54.1 \\
& & & \\
& & 73.9 \\
\hline
\end{tabular}


aged or non-evaluable cases were regarded as missing values. Score 0 was regarded as negative; scores 10 - 90 were regarded as low expression, $100-190$ as moderate, and $200-300$ as high expression.

Statistical analysis. Groups were compared using the the Mann Whitney U test and correlations were assessed by the Spearman's rank test. Cox proportion hazards regression model was used to assess the prognostic value of filamin A protein. Cox regression al and Kaplan-Meier analysis of survival was used to evaluate the relationship between filamin A protein expression and survival parameters in groups with different treatments (surgical operation + pt-based chemotherapy vs. surgical operation only). $\mathrm{P}<0.05$ was considered statistically significant. All tests were performed using SPSS softvare version 22 .

\section{Results}

Immunohistochemical expression of filamin A was characterized with marked variability in NSCLC, as well as in lung tissue surrounding the tumor. Bronchial columnar epithelial cells exhibited mainly moderate apical cytoplasmic and membranous staining, and relatively intensive staining in the attachment to the basal membrane. Submucosal glandular cells exhibited weak membranous positivity (Figure 1A). Bronchiolar connective tissue was either negative for filamin

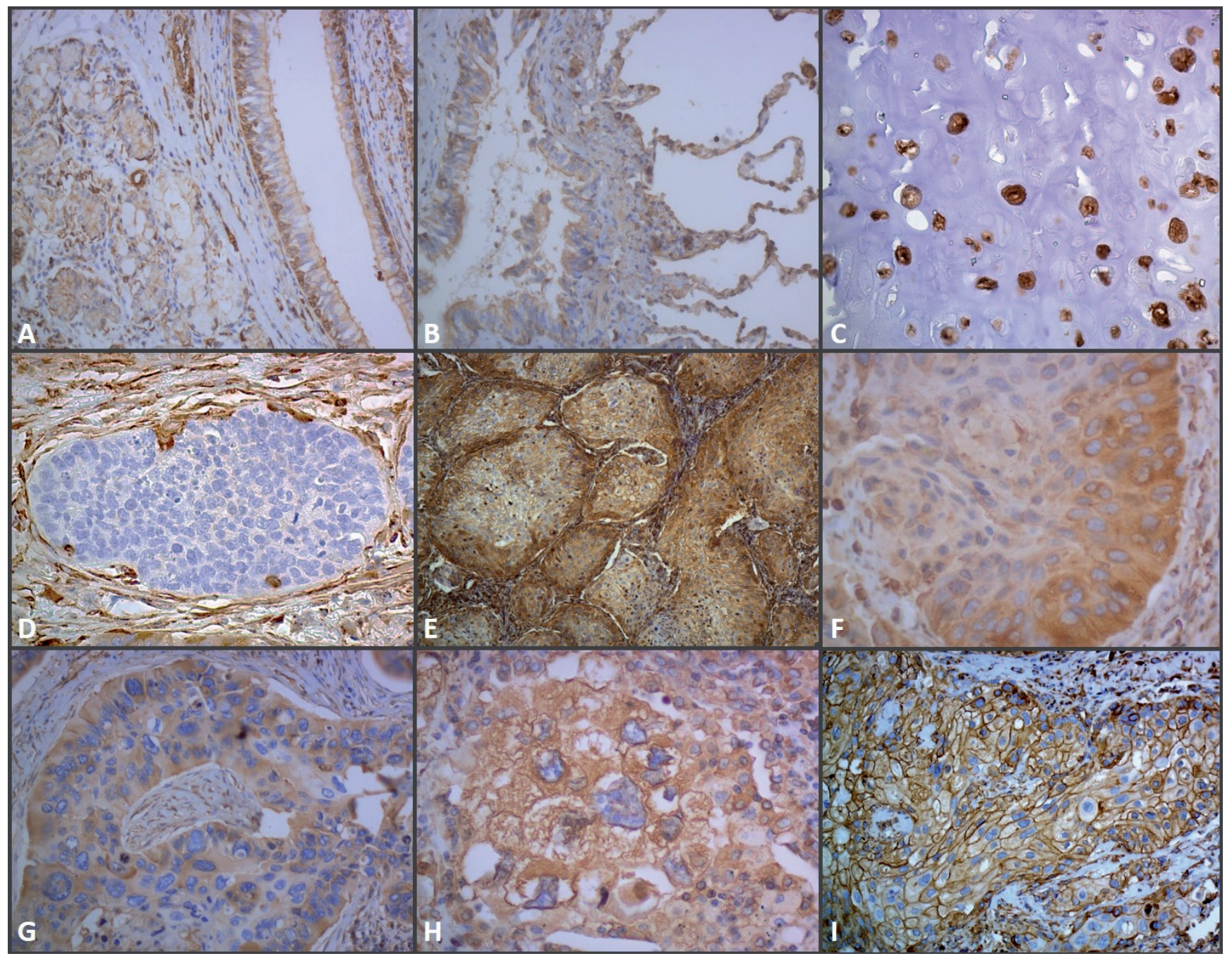

Figure 1. Filamin A protein expression in NSCLC and lung tissue surrounding the tumor. (A) weak to moderate staining of filamin A in the apical parts of bronchial epithelial cells and intensive staining in the attachement to basal membrane, weak membranous staining in submucosal glands (x200); (B) weak to moderate cytoplasmic and membranous expression in type I pneumocytes (x200); (C) strong expression in bronchiolar cartilage (x400); (D) moderate to strong filamin A expression in tumor stroma, which can be served as inner positive control for filamin A negative tumors (x200); (E,F) the increased expression of filamin A protein in the periferal parts of tumor tissue compared to the center of the tumor in SCC (x200, x400); (G) moderate cytoplasmic expression of filamin A in ADC (x200); (F) strong membranous and cytoplasmic expression of filamin Ain LCC (x400); (I) strong membranous expression of filamin A in SCC (x200). ( ${ }^{*}$ ADC- Adenocarcinoma, LCC - Large Cell Carcinoma SCC - Squamouc Cell Carcinoma) 
A or exhibited a weak cytoplasmic expression (Figure 1A,B). Chondrocytes in bronchiolar cartilage were strongly positive (Figure 1C). Type I pneumocytes showed weak to moderate cytoplasmic and membranous positivity (Figure 1B). NSCLC tissue showed weak to strong cytoplasmic and/or membranous expression of filamin A with marked intratumoral variability. Nuclear staining was present only in 6 cases. Tumor stroma was always moderately or strongly positive (Figure 1D) and was served as internal positive control for filamin A negative tumors. Filamin A expression was relatively high in the peripheral parts of tumor tissue, compared to central parts of solid tumors (Figure 1E,F). There was no significant difference in filamin A expression between different histological subtypes of NSCLC (Figure 3A). Some typical staining patterns are given in Figure 1 (G-I). IgG controls and matched NSCLC tissue staining are given in Figure 2.

After exclusion of non-representative, damaged or nonevaluable cases, 99 NSCLC patients were included in final statistical analysis. The comparison of filamin A expression in different clinico-pathological groups showed that, the highest expression of filamin A is characteristic for grade 2 tumors, followed by grade 3 and grade 1 tumors ( $<<0.05$, Figure $3 \mathrm{~B}$ ). Filamin A expression was positively associated with tumor size and invasion $(\mathrm{T})$, particularly it was significantly higher in T3-4 tumors, compared to T1-2 tumors ( $<<0.01$, Figure 3 C). Also, filamin A expression was higher in patients with lymph node $(\mathrm{N})$ and distant metastases $(\mathrm{M})(\mathrm{p}<0.05$, Figure $3 \mathrm{E}, \mathrm{F})$. Overall, filamin A cytoplasmic and membranous expression was significantly higher in stage IV disease, followed by stage III and stage I-II $(\mathrm{p}<0.01$, Figure $3 \mathrm{D})$. Spearman's rank test also showed a significant positive correlation between filamin A expression and NSCLC stage $(r=0.249 ; \mathrm{P}<0.05)$, lymph node metastases $(\mathrm{N})(\mathrm{r}=0.205 ; \mathrm{P}<0.05)$ and distant metastases $(\mathrm{M})$ $(\mathrm{r}=0.332 ; \mathrm{P}<0.01)$.

Based on univariate Cox proportional hazards regression model, patients within our cohort were divided into low and high risk groups according to filamin A expression, $<=90$ and $>90$ respectively. Particularly, the risk of tumor recurrence was significantly higher in patients with high $(>90)$ filamin A expression, compared to patients with low $(<=90)$ expression, despite the treatment $(\mathrm{HR}=1.00395 \% \mathrm{CI}[1.000: 1.005]$, $\mathrm{P}=0.02$, Figure $4 . \mathrm{B}$ ). The risk of death was also higher in patients with $>90$ filamin A expression $(\mathrm{HR}=1.003$ 95\%CI [1.000:1.005], $\mathrm{P}=0.07$, Figure 4). Multivariate Cox regression analysis showed that increased expression of filamin $\mathrm{A}(>90)$, represented an additional risk factor for NSCLC recurrence $(\mathrm{HR}=1.723,95 \% \mathrm{CI}$ [1.021:2.909], $\mathrm{P}<0.05)$, together with disease stage, tumor size, and the presence of lymph node metastasis.

Cox regression analysis of survival in different treatment groups showed that the increased expression of filamin A ( $>90)$ in patients, treated with platinum-based combination chemotherapy was significantly associated with lower $\mathrm{OS}$ rate $(\mathrm{HR}=1.005,95 \% \mathrm{CI}[1.000 ; 1.010], \mathrm{P}=0.037)$, as well as with DFS (HR=1.004, 95\%CI [1.001:1.008], $\mathrm{P}=0.017)$, whilst such association was not seen in patients treated with only surgical operation. The results of survival analysis plotted on Kaplan-Meier curves are shown on Figure 5. The quantitative distribution of filamin A expression in different clinicopathological groups is given in Table 2 .

\section{Discussion}

We found that filamin A expression is characterised by marked inter and intratumor variability in NSCLC. The strongest expression was observed in the peripheral parts of solid tumors and the expression of filamin A significantly correlated with primary tumor size and, the presense of lymph node and distant metastases. These findings suggest an important role of filamin A in NSCLC growth, invasion and metastases. Evidence for the role of filamin A in tumor progression is controversial in published literature. Some studies report that inhibition of filamin-A reduces cancer metastatic potential,

Table 2. Distribution of Filamin A expression in different clinico-pathological groups.

\begin{tabular}{|c|c|c|c|c|}
\hline & \multicolumn{2}{|c|}{ FILAMIN A HISTOSCORE } & \multirow[t]{2}{*}{ TOTAL } \\
\hline & & $<=90$ & $>90$ & \\
\hline \multirow{2}{*}{ EVENT OF DFS } & 0 & 22 & 6 & 28 \\
\hline & 1 & 35 & 36 & 71 \\
\hline \multirow{2}{*}{ EVENT OF OS } & 0 & 27 & 11 & 38 \\
\hline & 1 & 30 & 31 & 61 \\
\hline \multirow{3}{*}{ GRADE } & 1 & 8 & 4 & 10 \\
\hline & 2 & 13 & 16 & 27 \\
\hline & 3 & 37 & 21 & 58 \\
\hline \multirow{2}{*}{ ADJUVANT CHT } & 0 & 15 & 25 & 40 \\
\hline & 1 & 42 & 17 & 59 \\
\hline \multirow{4}{*}{ TNM STAGE } & 1 & 25 & 14 & 39 \\
\hline & 2 & 16 & 8 & 24 \\
\hline & 3 & 16 & 14 & 30 \\
\hline & 4 & 0 & 6 & 6 \\
\hline \multirow{5}{*}{$\mathbf{T}$} & 0 & 2 & 6 & 8 \\
\hline & 1 & 8 & 6 & 14 \\
\hline & 2 & 38 & 18 & 56 \\
\hline & 3 & 6 & 9 & 15 \\
\hline & 4 & 3 & 3 & 6 \\
\hline \multirow{4}{*}{$\mathbf{N}$} & 0 & 29 & 19 & 48 \\
\hline & 1 & 16 & 9 & 25 \\
\hline & 2 & 12 & 13 & 25 \\
\hline & 3 & 0 & 1 & 1 \\
\hline \multirow{2}{*}{$\mathbf{M}$} & 0 & 92 & 0 & 92 \\
\hline & 1 & 0 & 7 & 7 \\
\hline \multirow{3}{*}{ HISTOLOGY } & $\mathrm{ADC}$ & 19 & 17 & 36 \\
\hline & LCC & 7 & 5 & 12 \\
\hline & SCC & 32 & 19 & 51 \\
\hline
\end{tabular}




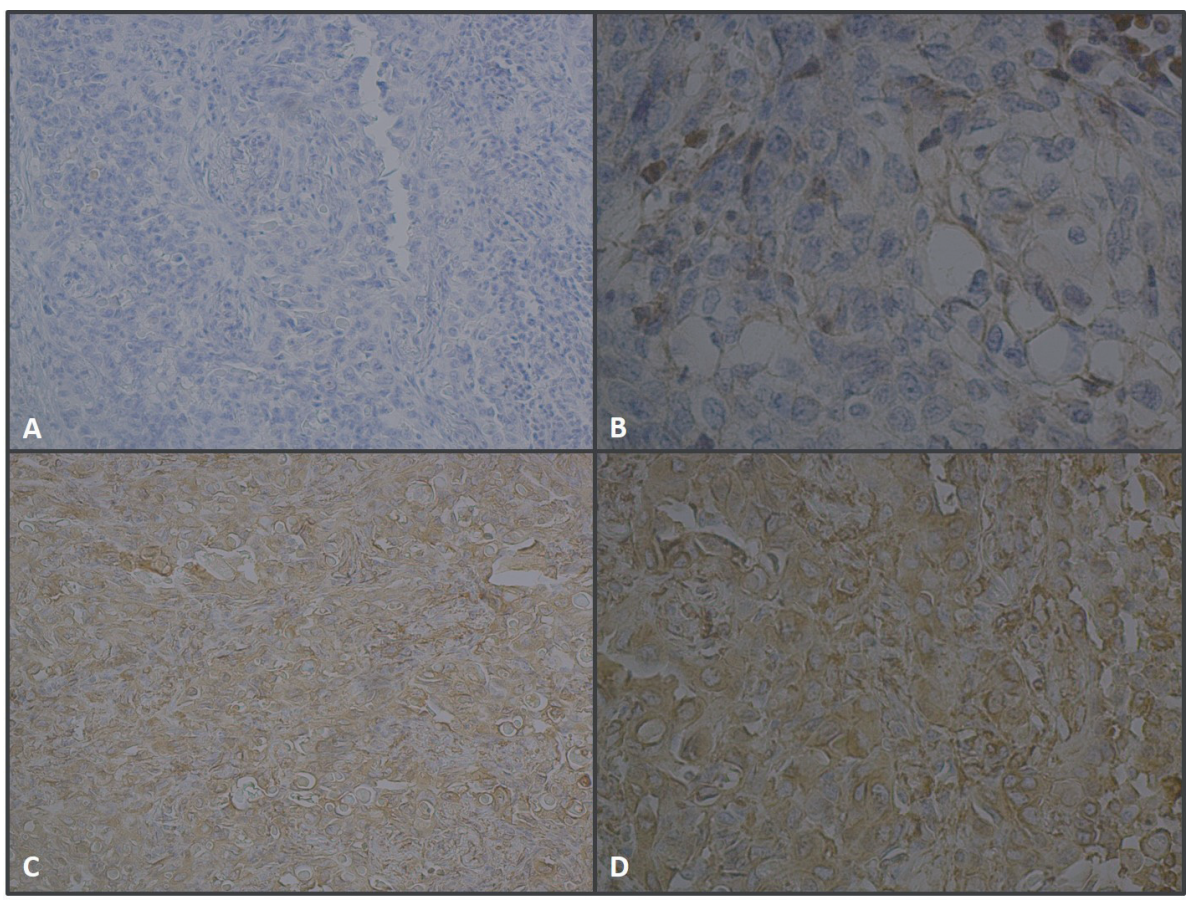

Figure 2. IgG control and filamin A staining of matched NSCLC tissue samples. (A) IgG control staining shows no reactivity (x200), whilst (C) the same tissue sample is positive for filamin A (x200); (B) IgG control staining shows some background staining (x400), clearly distinguishable from (D) specific filamin A positivity in the matched NSCLC tissue (x400).
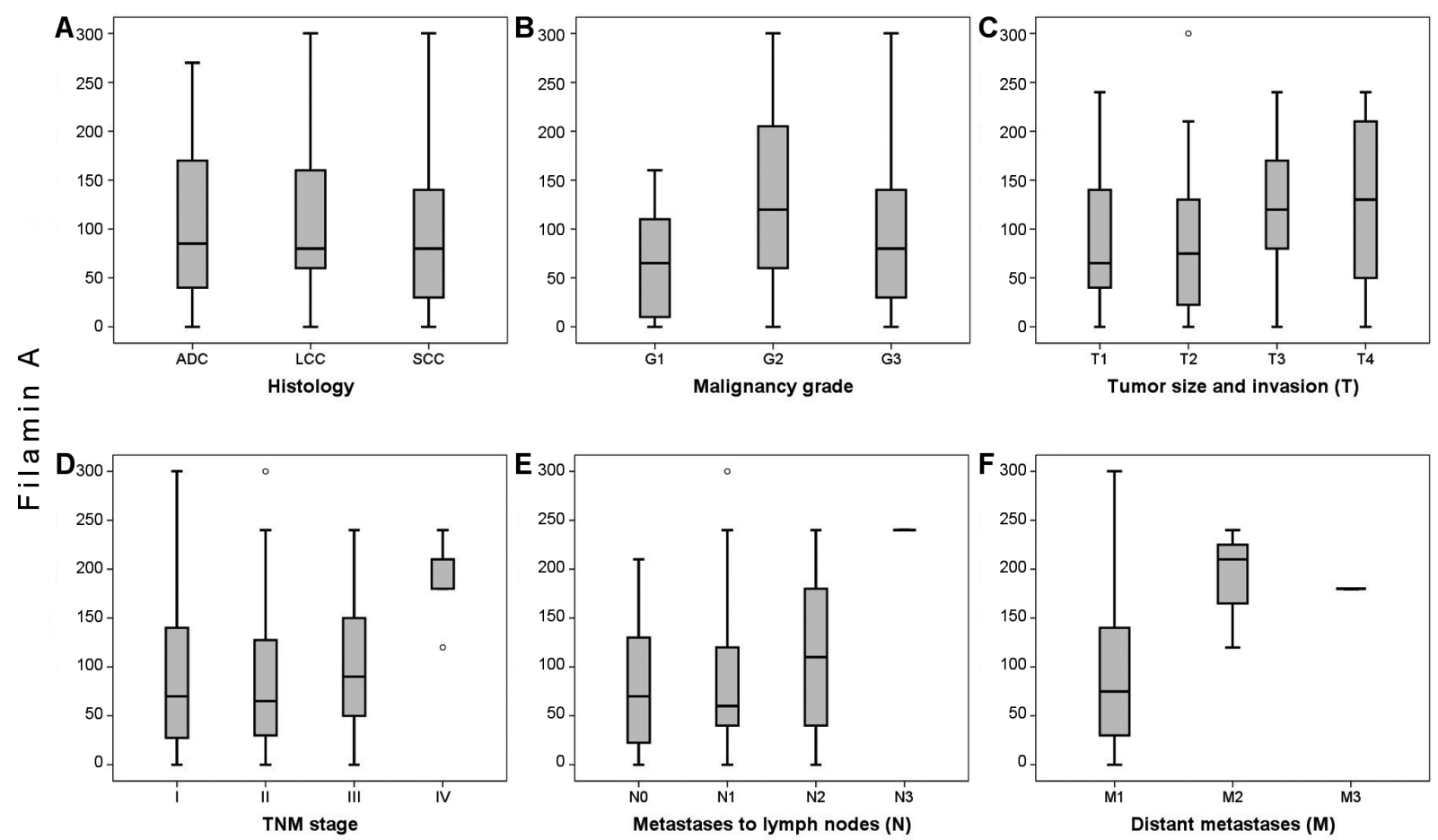

Figure 3. Distribution of filamin A protein expression in different histological and clinico-pathological groups of NSCLC (Mann-Whitney U). (A) There is no difference in filamin A expression, between different hitological subtypes (ADC - Adenocarcinoma, LCC - Large Cell Carcinoma, SCC - Squamous Cell Carcinoma); (B) The highest expression of filamin A is present in grade 2 tumors; (C) Filamin A expression is markedly higher in T3-4 tumors compared to T1-2; (D) The highes expression of filamin A is seen in stage IV NSCLC, followed by stage III and stage I-II; (E) Filamin A expression is higher in NSCLC patients with extensive metastatic spread (LN-lymph nodes) and (F) with the presense of distant metastases. 
whilst others show the opposite [reviewed by Yue et all., 2013] [5]. Our finding is supported by two previous experimental studies. Keshamouni et all. (2006) showed that filamin A protein is upregulated in A549 cells with high migratory potential [16]. Also, it has been shown that the targeting of filamin A results in significant decrease in size and invasive potential of lung cancer in mice [17]. In clincial studies, a positive relationship between overexpression of filamin-A protein and advanced stage, lymph node metastasis and vascular or neural invasion has been seen in breast and colorectal cancer patients [9], whilst the opposite was shown in nasopharingeal cancer [11]. This might be explained by the individual nature of each tumor.

Our second finding that filamin A protein represents an additional prognostic factor in NSCLC patients is supported by the multivariate Cox regression analysis, which showed that the overexpression of filamin A (>90) is a negative prognostic factor together with tumor size, disease stage and nodal status $(\mathrm{HR}=1.723,95 \% \mathrm{CI}$ [1.021:2.909], $\mathrm{P}<0.05)$. Similar findings were seen in colorectal cancer patients where

Hazard functions for Filamin A
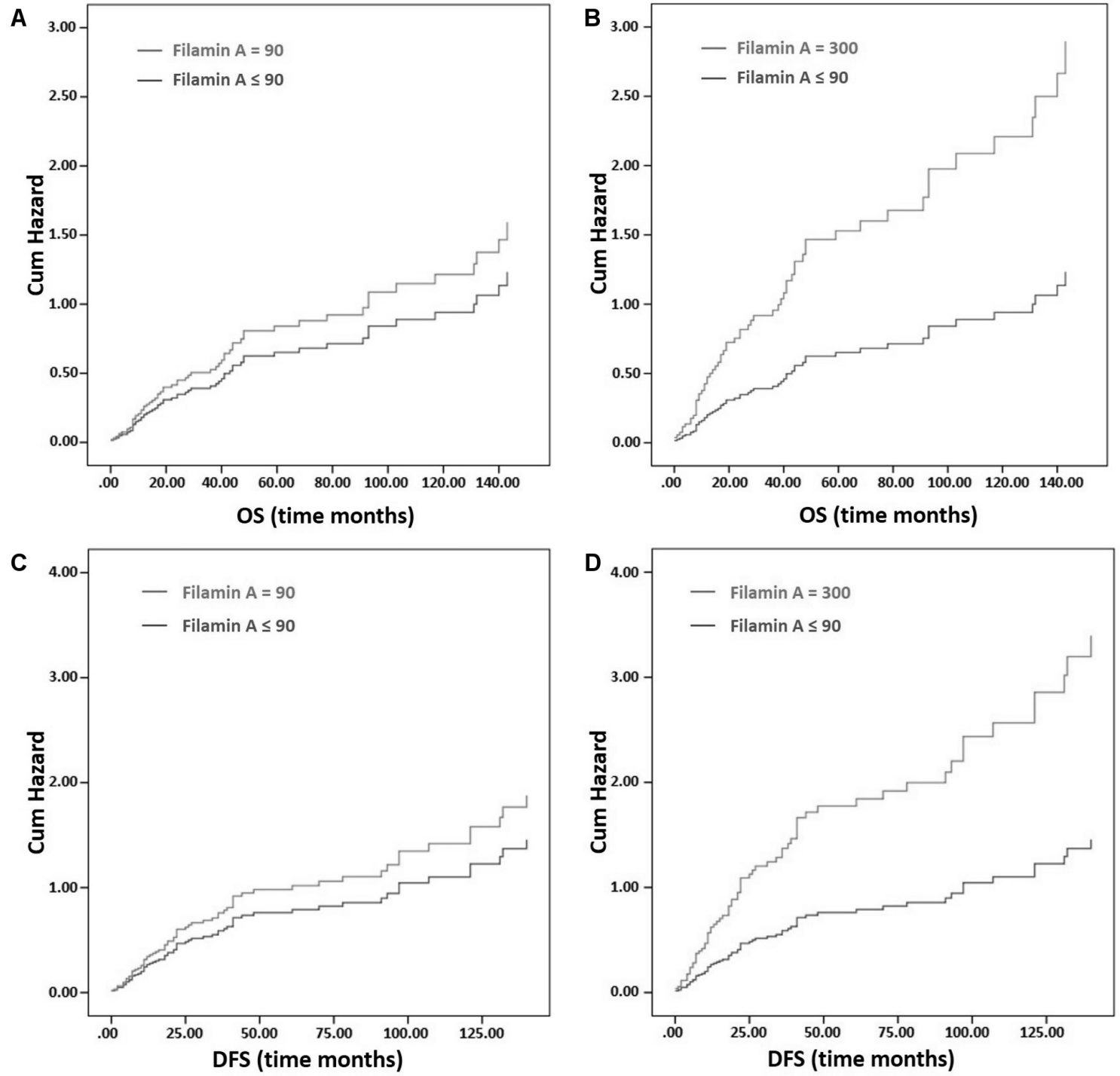

Figure 4. Cox regression hazard function and Kaplan-meier survival curves in relation to patient death and NSCLC relapse, calculated in months as overall (OS) and disease free survivals (DFS) respectively. (A) there is a slight difference in the hazard of patient death between lower levels of filamin A expression (histoscore $<\mathbf{9 0}$ and histoscore $=\mathbf{9 0}$ ), whilst $(\mathrm{B})$ the hazard of death is significantly increased in patients with high levels of filamin A (historcore $=300$ ), compared to patients with lower levels of filamin A (histoscore $<90)$; (C) the hazard of NSCLC relapse is slightly different at lower levels of filamin A expression (histoscore $<90$ and histoscore $=90$ ), whilst $(\mathrm{D})$ there is marked increase in the risk at higher expression $($ histoscore $=300$ ), compared to lower expression $(<90)$ of filamin $A(p<0.05)$. 
increased immunohistochemical expression of filamin A represented an independent prognostic factor together with lymph node metastases and depth of tumor invasion $(\mathrm{HR}=3.856,95 \% \mathrm{CI}[7.326: 19.421], \mathrm{P}<0.001)$. There has been only one study of filamin A protein expression in lung cancer patients, including small cell lung cancer (SCLC). This study suggested the possible role of filamin A in angiogenesis [18]. Similar to our study results these authors found that the overall 5-year survival rate for patients with positive and those with negative filamin A expression was $43.7 \%$ and $54.9 \%$, respectively $(p=0.06)$. However, univariate and multivariate survival analyses showed no relation with filamin A expression. This difference might be explained by the complexity of their study group and different evaluation method.
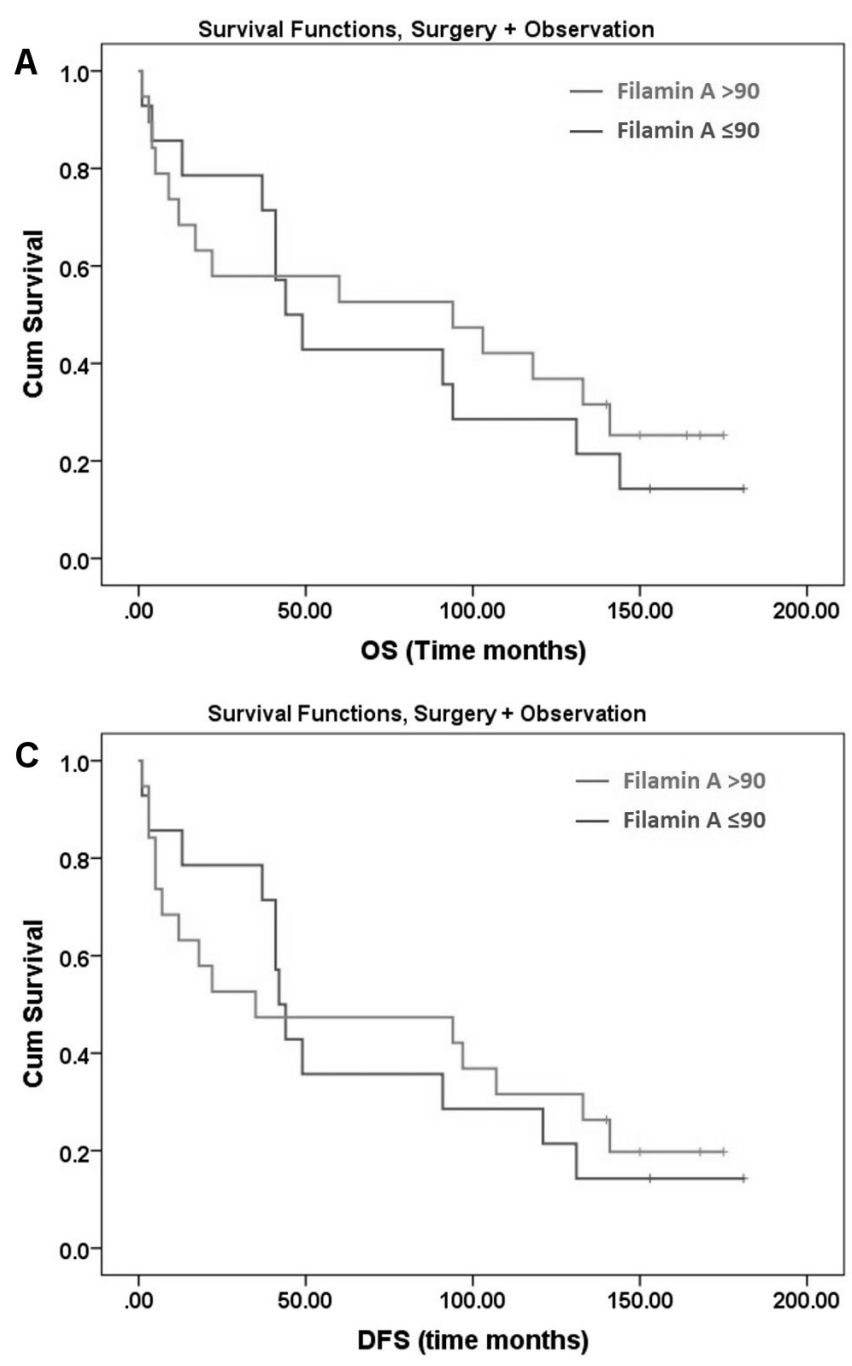

In presented study we stratified NSCLC patients into two treatment groups, those with and those without chemotherapy. Interestingly, there was a significant relationship between overexpression of filamin A and worst increased risk of relapse and death $((\mathrm{HR}=1.004,95 \% \mathrm{CI}[1.001: 1.008], \mathrm{P}=0.017)$ and $(\mathrm{HR}=1.005,95 \% \mathrm{CI}[1.000 ; 1.010], \mathrm{P}=0.037))$ in patients treated with carboplatine and navelbine, whilst such a relationship was not found for patients who underwent surgical treatment only. This finding further emphasizes the idea that filamin A plays a complex role not only in tumor growth and progression, but it also modulates the chemotherapy response, particularly in patients treated with platinum-based treatment regimens. To the best of our knowledge, this is the first clinical study to examine the relationship between filamin A expression and patient survival in two different treatment groups. However, the
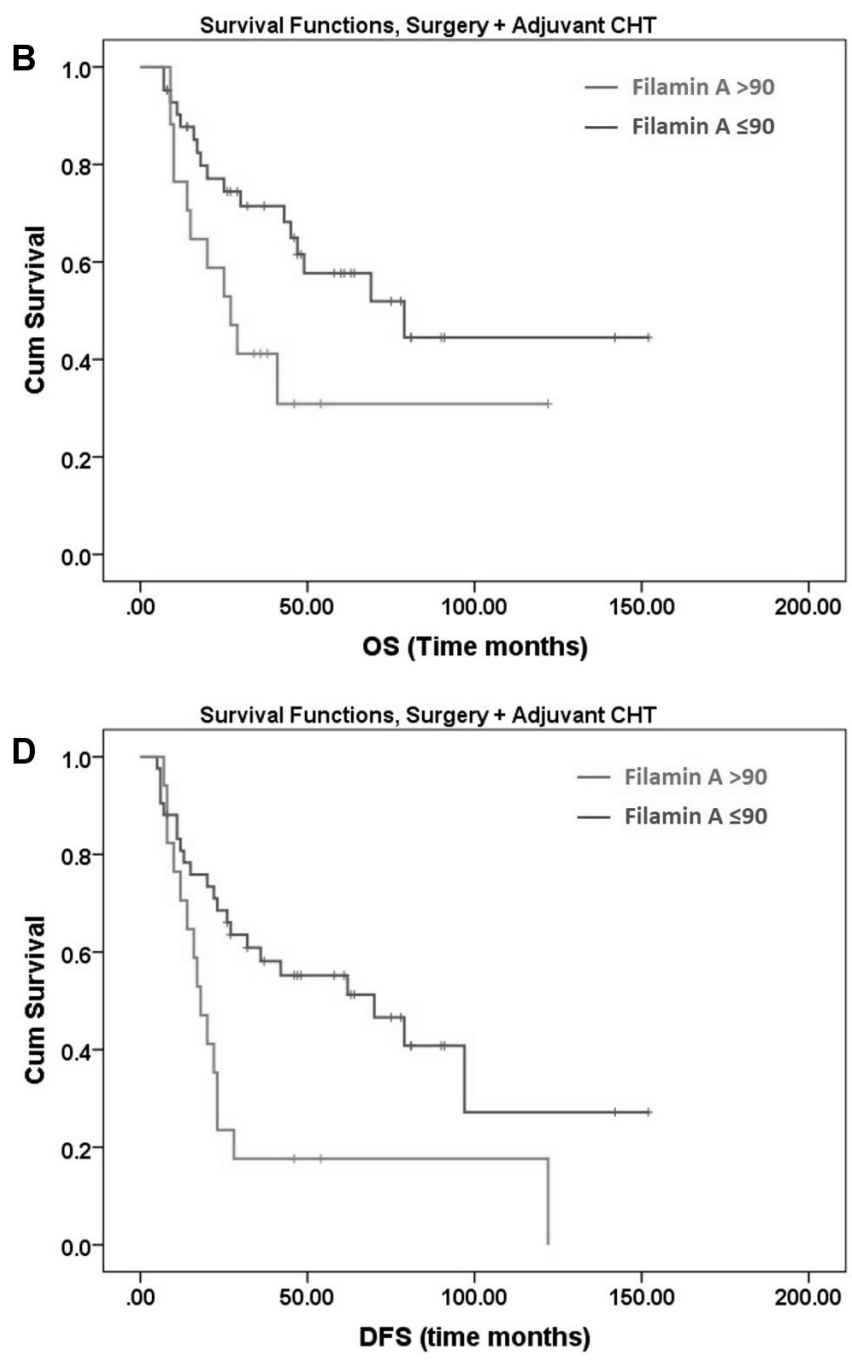

Figure 5. Kaplan-Meier survival curves in relation to filamin A expression and overall and disease free survivals (OS and DFS respectively) in different treatment groups. Figures A and C show that there is no difference in OS and DFS, based on filamin A expression in patients treated with only surgical operation; Figures B and D show that OS and DFS rates in patients with filamin A overexpression $(>90)$ is significantly lower compared to patients with low levels of filamin A expression $(<=90)(p<0.05)$. 
results are in accord with findings that suggest an important role of filamin A protein in DNA repair and resistance to cytotoxic drugs, including cisplatin $[14,17,18]$. Based on experimental study results on melanoma cell lines and mouse models, Yue et all. (2012) showed that not only endogenous levels filamin A reflect the chemosensitivity of tumor cells and not only it can help for the prediction of treatment response, but also filamin A might be used as a therapeutic target to sensitise cells to DNA damaging chemotherapy [14]. Our study also supports this finding, however more in vitro and in vivo experiments on NSCLC are necessary. Also, an extended imunohistochemical study with different filamin A antibodies would be desirable.

In conlcusion, our study results suggest that filamin A expression may represent an important prognostic marker for NSCLC progression and might help to predict platinum-based treatment response.

Acknowledgements: This publication was supported by grants NT/13569 from the Czech Ministry of Health and NPU I LO1304 from the Czech Ministry of Education. Mariam Gachechiladze was also supported by student's grant LF_2014_003.

\section{References}

[1] SIEGEL R, NAISHADHAM D, JEMAL A. Cancer statistics. CA: a cancer journal for clinicians 2012; 62: 10-29. http:// dx.doi.org/10.3322/caac. 20138

[2] PIGNON JP, TRIBODET H, SCAGLIOTTI V, DOUILLARD JV, SHEPARD FA et al. Lung adjuvant cisplatin evaluation: a pooled analysis by the LACE Collaborative Group. Journal of clinical oncology: official journal of the American Society of Clinical Oncology, 2008; 26: 3552-3559. http://dx.doi. org/10.1200/JCO.2007.13.9030

[3] ROSELL R, LORD RV, TARON M, REGUART N. DNA repair and cisplatin resistance in non-small-cell lung cancer. Lung cancer (Amsterdam, Netherlands),2002; 38: 217-227 http:// dx.doi.org/10.1016/S0169-5002(02)00224-6

[4] BONANNO L, FAVARETTO A, ROSELL R. Platinum drugs and DNA repair mechanisms in lung cancer. Anticancer research, 2014; 34: 493-501

[5] YUE J, HUHN S, SHEN Z. Complex roles of filamin-A mediated cytoskeleton network in cancer progression. Cell \& bioscience, 2013; 3: 7 http://dx.doi.org/10.1186/2045-3701$\underline{3-7}$

[6] NOTTERMAN DA, ALON U, SIREK AJ, LEVINE AJ. Transcriptional gene expression profiles of colorectal adenoma, adenocarcinoma, and normal tissue examined by oligonucleotide arrays. Cancer research, 2001; 61: 3124-3130

[7] LI C, YU S, NAKAMURA F, YIN S, XU J et al. Binding of proprion to filamin A disrupts cytoskeleton and correlates with poor prognosis in pancreatic cancer. The Journal of clinical investigation, 2009; 119: 2725-2736 http://dx.doi.org/10.1172/ LCI39542

[8] GUEDJ N, ZHAN Q, PERIGNY M, RAUTOU PE, DEGOS $\mathrm{F}$ et al. Comparative protein expression profiles of hilar and peripheral hepatic cholangiocarcinomas. Journal of hepatology, 2009; 51: 93-101 http://dx.doi.org/10.1016/j. jhep.2009.03.017

[9] TIAN HM, LIU XH, HAN W, ZHAO LL, YUAN B et al. Differential expression of filamin A and its clinical significance in breast cancer. Oncology Letters, 2013; 6: 681-686,

[10] SMITH SC, OXFORD G, BARAS AS, OWENS C, HAVALESHKO D et al. Expression of ral GTPases, their effectors, and activators in human bladder cancer. Clinical cancer research: an official journal of the American Association for Cancer Research, 2007; 13: 3803-3813 http://dx.doi. org/10.1158/1078-0432.CCR-06-2419

[11] SUN GG, LU YF, CHENG YJ, HU WN. Absent expression of FLNA is correlated with poor prognosis of nasopharyngeal cancer. Tumour biology: the journal of the International Society for Oncodevelopmental Biology and Medicine, 2014; 35, 2967-2974

[12] VELKOVA A, CARVALHO MA, JOHNSON JO, TAVTIGIAN SV, MONTEIRO AN. Identification of Filamin A as a BRCA1-interacting protein required for efficient DNA repair. Cell cycle, 2010; 9: 1421-1433 http://dx.doi.org/10.4161/ cc.9.7.11256

[13] YUAN Y, SHEN Z. Interaction with BRCA2 suggests a role for filamin-1 (hsFLNa) in DNA damage response. The Journal of biological chemistry, 2001; 276: 48318-48324

[14] YUE J, LU H, LIU J, BERWICK M, SHEN Z. Filamin-A as a marker and target for DNA damage based cancer therapy. DNA repair, 2012; 11: 192-200 http://dx.doi.org/10.1016/j. dnarep.2011.10.019

[15] PROFILES G. A2780 epithelial ovarian cancer cell line with acquired platinum resistance, Expression profiling by array. http: //www.ncbi.nlm.nih.gov/geoprofiles/66837447.

[16] KESHAMOUNI VG, MICHAILIDIS G, GRASSO CS, ANTHWAL S, STAHLER JR et al. Differential protein expression profiling by iTRAQ-2DLC-MS/MS of lung cancer cells undergoing epithelial-mesenchymal transition reveals a migratory/ invasive phenotype. Journal of proteome research, 2006; 5: 1143-1154 http://dx.doi.org/10.1021/pr050455t

[17] NALLAPALLI RK, IBRAHIM MX, ZHOU AX, BANDARU S, SUNKARA SN et al. Targeting filamin A reduces K-RASinduced lung adenocarcinomas and endothelial response to tumor growth in mice. Molecular cancer, 2012; 11: 50 http:// dx.doi.org/10.1186/1476-4598-11-50

[18] URAMOTO H, AKYUREK LM, HANAGARI TA. positive relationship between filamin and VEGF in patients with lung cancer. Anticancer research, 2010; 30: 3939-3944 